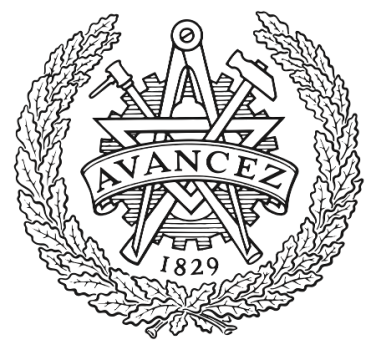

CHALMERS

UNIVERSITY OF TECHNOLOGY

\title{
Mathematical Definitions of Scene and Scenario for Analysis of Automated Driving Systems in Mixed-Traffic Simulations
}

Downloaded from: https://research.chalmers.se, 2023-04-26 13:45 UTC

Citation for the original published paper (version of record):

Andreotti, E., Boyraz Baykas, P., Selpi, S. (2021). Mathematical Definitions of Scene and Scenario for Analysis of Automated Driving Systems in

Mixed-Traffic Simulations. IEEE Transactions on Intelligent Vehicles, 6(2): 366-375.

http://dx.doi.org/10.1109/TIV.2020.3031981

N.B. When citing this work, cite the original published paper. 
Author's post-print accepted for publication in the IEEE Transactions on Intelligent Vehicles (Oct. 2020).

(C)2020 IEEE. Personal use of this material is permitted. Permission from IEEE must be obtained for all other users, including reprinting/ republishing this material for advertising or promotional purposes, creating new collective works for resale or redistribution to servers or lists, or reuse of any copyrighted components of this work in other works.

DOI: 10.1109/TIV.2020.3031981 


\title{
Mathematical Definitions of Scene and Scenario for Analysis of Automated Driving Systems in Mixed-Traffic Simulations
}

\author{
Eleonora Andreotti, Pinar Boyraz, and Selpi
}

\begin{abstract}
This paper introduces a unified mathematical definition for describing commonly used terms encountered in systematical analysis of automated driving systems in mixedtraffic simulations. The most significant contribution of this work is in translating the terms that are clarified previously in literature into a mathematical set and function based format. Our work can be seen as an incremental step towards further formalisation of Domain-Specific-Language (DSL) for scenario representation. We also extended the previous work in the literature to allow more complex scenarios by expanding the model-incompliant information using set-theory to represent the perception capacity of the road-user agents. With this dynamic perception definition, we also support interactive scenarios and are not limited to reactive and pre-defined agent behavior. Our main focus is to give a framework to represent realistic roaduser behavior to be used in simulation or computational tool to examine interaction patterns in mixed-traffic conditions. We believe that, by formalising the verbose definitions and extending the previous work in DSL, we can support automatic scenario generation and dynamic/evolving agent behavior models for simulating mixed traffic situations and scenarios. In addition, we can obtain scenarios that are realistic but also can represent rare-conditions that are difficult to extract from field-tests and real driving data repositories.
\end{abstract}

Index Terms-scene, scenario, situation, scenery, formal definitions, interactive agents, automated driving, mixed-traffic, traffic simulations

\section{INTRODUCTION}

There are two motivations driving the research efforts in definition of a formal language for describing the complex situations in mixed-traffic simulations: (i) Development of automated driving systems require extensive scenario testing before deployment and the common language allows different systems to be bench-marked on a fair manner, (ii) The coexistence of human-operated vehicles (HOV), autonomous vehicles (AV) and vulnerable road users (VRU) (i.e. bikers, cyclists, pedestrians, e-vehicle users) require the public authorities to examine complex scenarios for better traffic and transportation management as well as digital and physical infrastructure design. In fact, the milestone of such efforts has started with [1] in which scene, scenario and situation terms were clarified and their scope was determined. Building upon these clear definitions and separation between the scenario and

\footnotetext{
*E. Andreotti, P. Boyraz and Selpi are with the Division of Vehicle Safety, Department of Mechanics and Maritime Sciences, Chalmers University of Technology, SE-412 96 Göteborg, Sweden

1 eleonora.andreotti@chalmers.se

${ }^{2}$ pinar.boyraz@chalmers.se

3 selpiechalmers.se
}

situation, [2] developed a Domain-Specific-Language (DSL), named 'GeoScenario' to be used in scenario representation to substantiate test cases for automated driving systems (ADS). GeoScenario is a perfect DSL for providing ways of reproducing trajectory of road-user agents and also carefully designed mechanisms of coordinating their actions (Please see Section $\mathrm{C}$, last paragraph in [2]). However, in its current state, to the best of authors' knowledge, it cannot provide complete and realistic intelligent behavior of road-user agents, featuring dynamic models of road-user agents, yet. In addition to this, this DSL does not specify details of vehicle model dynamics, therefore may not shed light in terms of individual vehicle parameters and their effect in microscopic traffic simulations. For example, the avoidance manoeuvres cannot be fully represented with their exact trajectories in such platforms at this scale. Next to the GeoScenario, another approach for the scenario creation is based on computational ontology, Studer et al. [3]. Guarino et al. [4] make use of set theory to provide a more precise and formal view of the aspects of Studer's definition of computational ontology. Bagschik et al. [5] also propose a process for a computational ontology based scene creation for the development of Automated Vehicles, subsequently adapted to the ontology based scenario creation [6]. This approach uses a 5-layer-model, expanding a 4-layer-model proposed by Schuldt et al. [7], combined with Ulbrich's scene definition [1]. Each entity (represented by a word) within a layer represents multiple relations to parameters in the physical state space and to represent interactions of layers the authors propose to annotate if an entity includes or influences a parameter. This approach, proposed for automated vehicles scene, limits the interaction between objects at the given instant of the scene and does not consider that every "thinking entity" (automated or not) of the scene makes its own projections about how it expects the other entities to behave in the near future, and that its reactions are based on its expectations. Beside that, a connection between the functional scenario (described in a linguistic way) and the logical (made up of parameters and parameter ranges) and concrete scenarios (composed by concrete value for each parameter) is necessary [8], [9]. There are also great efforts in designing Open simulation platforms, which can enable multi-agent simulations to estimate the impact of ADS with a focus on safety. In [10], such a platform is used to see the effect of penetration of SAE Level 1 and 2 technologies, such as automated emergency braking (AEB) and lane departure warning (LDW), on safety. It was also possible to demonstrate how real road-network and field 
data from reported accidents can be combined to obtain a larger assessment of ADS. Although, they employ an advanced platform to simulate this large-scale mixed-traffic with multiagent approach, they do not dwell much on the scenario and situation definitions and the software structure is not given in detail yet. Another important aspect of agent-based mixedtraffic simulations is their level of rigour when defining trafficflow or safety metrics to identify potential problems related to congestion and traffic safety. In order to represent these aspects in a quantitative and holistic way, [11] has borrowed the 'potential field' approach that is used to solve the problem of path-planning in robotics, avoiding static or dynamic obstacles. In that work, concept of potential field was extended to include fields of risk from vehicle's motion, driver's perception and environmental factors. In order to include the perceptions of agents, several efforts have been made also in SUMO and MITSIMLab [12], [13]. While, in [14], the agents perform tactical-level driving and the manoeuvres performed in every situation are decided in real time. However, the forces are not modeled and the consequences of weather, for example, cannot be taken into account, e.g. the vehicles will perform manoeuvres without slipping. The most recent efforts in the microscopic traffic simulation's field are well summarized in [15].

In this paper, we propose mathematical definitions of scene, scenario, and situation, using the verbal definitions proposed by [1] as a starting point. Due to our mathematical definitions, we can create a link between spoken language and mathematical models, which can be used to expand the already existing micro-scale traffic simulations. In this context, the set theory is useful to identify objects sharing common properties, and thereby grouping them together.

In doing so, we extend the model-incompliant information to represent the perception capacity of the road-users agents, i.e. the awareness of things through the physical senses. The perception of the road-user can be human perception (human road-user), machine perception (i.e. for autonomous vehicle), or a mixture of both. Therefore, these perceptions depend on the presence/absence and manufactured functionality of the vehicle's sensors, on the driver emotional state (gloomy/happy, worried/carefree, nervous/calm, bored/interested, angry/peaceful) and on driver personality type (lazy/dynamic, irresolute/resolute, impatient/patient, impulsive/reflective, superficial/meticulous) and driver physical condition (tired/refreshed, sleepy/awake, sick/healthy), see [16], [17] for detailed information. In [18], it is shown that the $90-95 \%$ of accidents are caused by human errors in information processing and inattention/lack of attention, while in [19] the authors highlighted that the more experienced drivers have a better understanding of other road users' communicative signals and are more quickly to detect road hazards.

Our paper is organized as follows: in Sections II, III and IV, we respectively introduce the mathematical definitions of scene, situation and scenario, and illustrate these definitions using examples. In Section V, we show how our definitions could be used to describe a scenario, and the scenario from each road-users' different point-of-view, therefore extending the perception component in scenario representation. We have compiled discussion points in Section VI to identify unresolved problems and set path for future work in evaluation of ADS using multi-agent traffic simulations. Finally, conclusions are drawn in Section VII.

\section{DEFINING THE TERM SCENE}

In order to define the term scene, let's start by defining some preliminary concepts that are useful for our purpose. Let us start by considering a portion of space, $\mathcal{S}$, in the real world. We define the static object set $\mathcal{V}^{s}(\mathcal{S})$ as the set of the whole motionless objects (lane network, stationary elements, vertical elevation) which are located in the portion of space $\mathcal{S}$. By motionless objects, we mean those objects that stand still for a sufficiently long time, or whose movements are almost imperceptible.

Example 1. Static object set, $\mathcal{V}^{s}(\mathcal{S})$

The portion of space $\mathcal{S}$, in the real world, can be represented as the region delimited by the black perimeter in Fig.la. In order to represent the static object set of the portion of space $\mathcal{S}, \mathcal{V}^{s}(\mathcal{S})$, we should delete from the picture all the dynamic elements, i.e. all the objects in Fig.1a whose position changes as a function of time. In Fig. $1 b$ we removed the vehicle and bicycles, thus we get the set of the whole motionless road-objects be located in the portion of space $\mathcal{S}$, i.e. the static object set.

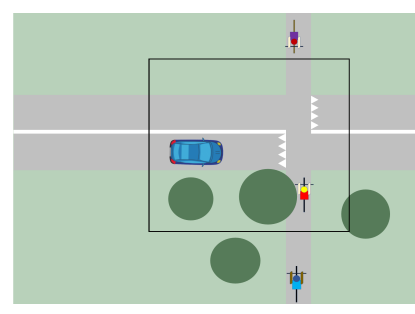

(a)

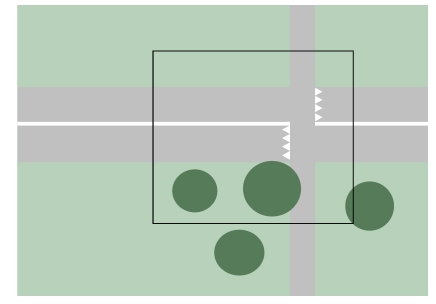

(b)
Fig. 1: Real (a) and static (b) object set representations of a portion of space $\mathcal{S}$ (delimited by the black perimeter).

Let $\mathcal{V}^{d}(\mathcal{S}, t)$ be the set of the whole dynamic elements (i.e. pedestrians, cyclists, HOVS, AVs) be located in the portion of space $\mathcal{S}$ at time $t$. Each element of the $\operatorname{set} \mathcal{V}^{d}(\mathcal{S}, t)$ is called actor. The union of the sets $\mathcal{V}^{d}(\mathcal{S}, t)$ and $\mathcal{V}^{s}(\mathcal{S})$ is denoted as $\mathcal{V}(\mathcal{S}, t):=\mathcal{V}^{s}(\mathcal{S}) \cup \mathcal{V}^{d}(\mathcal{S}, t)$ and called the object set. Each element of $\mathcal{V}(\mathcal{S}, t)$ is called object. Throughout the paper, when there is no scope for ambiguity, we omit the letter $\mathcal{S}$ and $t$ from set's symbols and write, for example, $\mathcal{V}^{s}$ and $\mathcal{V}^{d}$ instead of $\mathcal{V}^{s}(\mathcal{S})$ and $\mathcal{V}^{d}(\mathcal{S}, t)$.

In [1, p. 983], the authors define a scene as "a snapshot of the environment including the scenery and dynamic elements, as well as all actors' and observers' self representations, and the relationships among those entities". Until now, we have defined the object set which includes all the static and dynamic elements. In order to define a scene we need to define "the scenery" as well as "the relationships among those entities" and "all actors' and observers' self representations", and to achieve this, it is useful to introduce a position function. Typically $\mathcal{S}$ can be a portion of space given in geographical coordinate system, or in any other coordinate system, and the function $\mathcal{C}: \mathcal{V}(\mathcal{S}, t) \rightarrow \mathcal{S}$ associate each 
object in $\mathcal{V}(\mathcal{S}, t)$ to its dimensional coordinates in the coordinate system of $\mathcal{S}$, Fig.2.

Let $x \in \mathcal{V}(\mathcal{S}, t)$ then

$$
\mathcal{C}: \mathcal{V}(\mathcal{S}, t) \rightarrow \mathcal{S}, \quad x \mapsto \mathcal{C}(x)
$$

From function (1) we can derive the surface occupied by the object $x$ and the mutual relations among two or more objects. This function $\mathcal{C}$ is called position function and the set $\mathcal{C}(\mathcal{V})$ is the position set. Sometimes it is useful to write $x(t)$ to mean that we are considering the object $x$ at time $t$, and then through $\mathcal{C}$ we associate $x$ to its position at time $t$.

\section{Example 2. Position set}

Each object $x \in \mathcal{V}$ is associated, through function $\mathcal{C}$, to its dimensional coordinate in $\mathcal{S}$. For instance, in the example shown in Fig.2, each $x$ can be associated to coordinates of 4 points, $P, P^{\prime}, P^{\prime \prime}$ and $P^{\prime \prime \prime}$ (in a 2-dimensional space $\mathcal{S})$ which represent the vertices of the minimum quadrangle that contains the object $x$, i.e. $\mathcal{C}: \mathcal{V} \rightarrow \mathcal{S}, x \mapsto \mathcal{C}(x)=$ $\left\{P, P^{\prime}, P^{\prime \prime}, P^{\prime \prime \prime}\right\}$. To be more general we could consider polygons with more than 4 vertices.

Remark 1. The vertices in Example 2 delimit the occupancy area. We could work in 3 dimensions, and in such a case we would have the vertices (by extending the quadrangle we could consider parallelepipeds) to represent the occupancy volume.

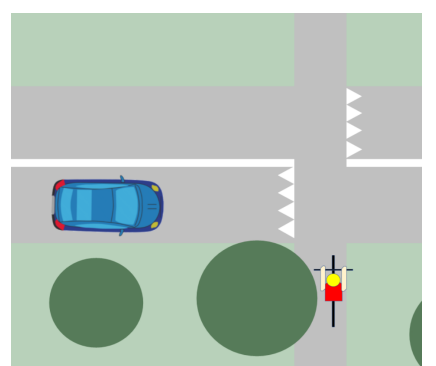

(a)

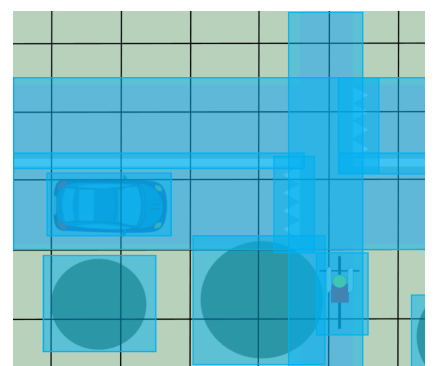

(b)
Fig. 2: Position set $\mathcal{C}(\mathcal{V})$. (a) Set $\mathcal{V}$ of the portion of space $\mathcal{S}$ at time $t$. (b) Set $\mathcal{V}$ in dimensional coordinate representation $\mathcal{C}(\mathcal{V})$, i.e. the position set.

The couple $(\mathcal{V}, \mathcal{C})$ provides us the real (objective, ground truth) snapshot of the portion of space $\mathcal{S}$ and the objects contained in it at time $t$. However each actor $x$ (pedestrian, cyclist, HOV, AV, etc.) may not see and perceive the whole information contained in the couple $(\mathcal{V}, \mathcal{C})$. Each actor has a role as an observer and within that role has its own representation (i.e. what it can see or perceive).

Moreover, the observer's perception may depend on what type of observer it is, and from the moment in which it finds itself. For example an automated vehicle (AV) observer will have different perceptions than an elderly driver of a non-automated vehicle observer or an observer who checks the status of road from navigation system. Consistent with the previous definitions, we define the object set from $x$ 's point of view, or $x$-object set, $\mathcal{V}_{x}$, as the subset of $\mathcal{V}$ of the objects that $x$ sees or perceives at time $t$, Fig.3.
Example 3. Object set from $x$ 's point of view, $\mathcal{V}_{x}$ Fig.3 represents the object sets from car $(x)$ point of view, $\mathcal{V}_{x}$, and from bicycle $(y)$ point of view, $\mathcal{V}_{y}$. Using Example 1 we get $\mathcal{V}_{x} \neq \mathcal{V}$ and $\mathcal{V}_{x} \neq \mathcal{V}_{y}$ and this is due to the fact that car is not able to see the bicycle because of the presence of the tree.

Aligned with the previous definitions and in order to define a

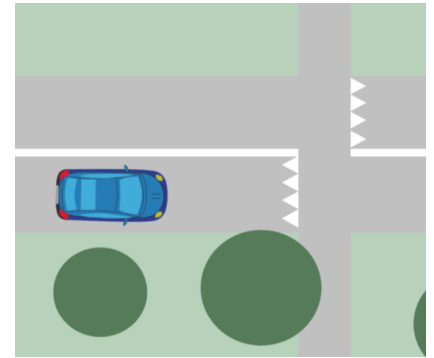

(a)

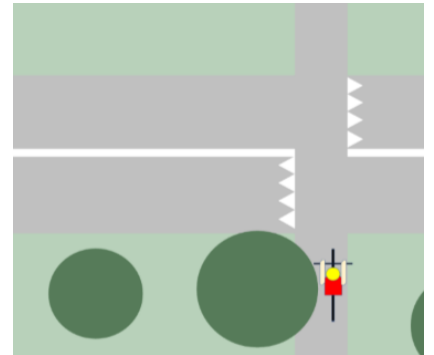

(b)
Fig. 3: Object sets from car's driver (a) and cyclist (b) point of view, $\mathcal{V}_{x}$ and $\mathcal{V}_{y}$ respectively.

scene it turns out to be crucial to define the position function from $x$ 's point of view. We consider the $x$ 's point of view in the coordinate system of $\mathcal{S}$ : we define $\mathcal{C}_{x}$ as the position from $x$ 's point of view function, or $x$-position function as follows:

$$
\mathcal{C}_{x}: \mathcal{V}_{x} \rightarrow \mathcal{S}, \quad y \mapsto \mathcal{C}_{x}(y)
$$

Sometimes it is useful to make the time dependence of $y$ explicit and write $y(t)$ to mean that we are considering the object $y$ at time $t$, and then through $\mathcal{C}_{x}$ we associate $y$ to its position at time $t$. The image set of $\mathcal{V}_{x}$ under the $x$-position function, $\mathcal{C}_{x}\left(\mathcal{V}_{x}\right)$, is called position from $x$ 's point of view set, or $x$-position set for brevity. In general it is not true that $y \in \mathcal{V}_{x} \subset \mathcal{V} \Rightarrow \mathcal{C}_{x}(y)=\mathcal{C}(y)$. Thus, even if an object $y$ can be seen or perceived from the ego-vehicle $x$ it does not necessarily mean that $x$ has an objective view of $y$ position or size (for example perception loss due to faulty or malfunctioning sensors); see illustrations in Fig.4 and Example 4.

\section{Example 4. Model-incompliant information}

In scene definition, the static and dynamic model incompliant information are implicitly taken into consideration. Indeed, for instance, the set $\mathcal{V}_{x}$ collect all the objects that $x$ can see and perceive, but this doesn't mean that $x$ really sees or perceives them. The "cognitive" aspect of the model and, more generally, the model incompliant information are represented by the function $\mathcal{C}_{x}$. Let us suppose to be in the case described in Fig.(3), where the car driver cannot see or perceive the cyclist. Then we get an $x$-position set $\mathcal{C}_{x}\left(\mathcal{V}_{x}\right)$ which does not include the bike. Now let us suppose the bike is coming from the left of the car (Fig.4b). In this case the car driver is supposed to be able to see it, but for some reasons the car driver is not able to see it, due to e.g. failure of sensors for automated vehicles $(A V)$ or inattentive driver for human-operated vehicles (HOV). In this case the bike belongs to $\mathcal{V}_{x}$ but the function $\mathcal{C}_{x}$ associates an empty set to it.

So far, we have defined the set of objects and the function 


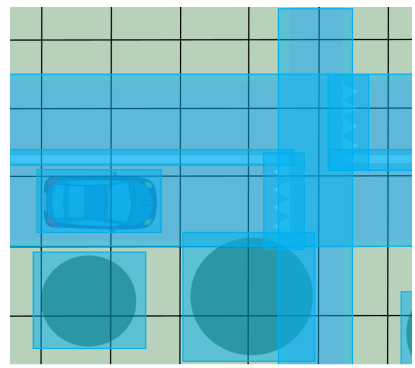

(a)

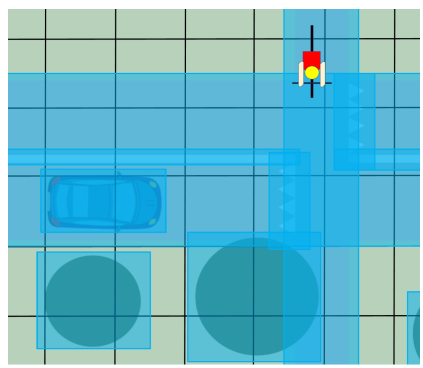

(b)
Fig. 4: Two different cases that give the same sets of position from $x$ (car) point of view, $\mathcal{C}_{x}\left(\mathcal{V}_{x}\right)$. (a) Using Example 1 to illustrate the case, the cyclist $y$ under the tree is not represented because $x$ is not able to see it, in this case the cyclist $y \in \mathcal{V}$ but $y \notin \mathcal{V}_{x}$. (b) In this second example a cyclist $\tilde{y}$ (top right) is represented because $x$ is supposed to be able to see it, $\tilde{y} \in \mathcal{V}_{x}$, but $\mathcal{C}_{x}(\tilde{y}) \neq \mathcal{C}(\tilde{y})$.

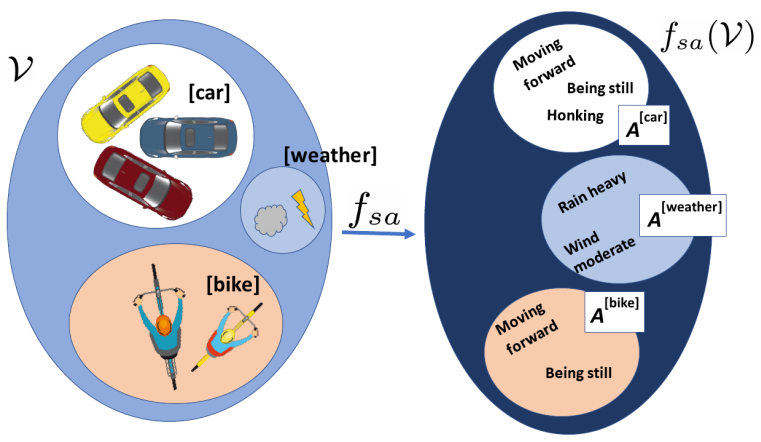

Fig. 5: States \& attributes function.

that defines their positions, both from the point of view of an omniscient observer and from the point of view of any observer. Now we are going to define an equivalence relation in order to categorise the objects within the set of objects. Indeed, we cannot expect pedestrian objects to have the same behavior or characteristics as cyclist objects, as well as HOV objects with AV objects.

Let us define $\sim$ the equivalence relation as "is the same type of object", and $V$ (or $V_{x}$ ) the quotient sets of $\mathcal{V}$ (or $\mathcal{V}_{x}$ ) by $\sim$, i.e. the set of all possible equivalence classes of $\mathcal{V}$ (or $\mathcal{V}_{x}$ ) by $\sim$. Let $[x]:=\{y \in \mathcal{V} \mid x \sim y\}$ denote the equivalence class to which $x$ belongs. All elements of $\mathcal{V}$ equivalent to each other are also elements of the same equivalence class.

Remark 2. The equivalence relation $\sim$ can be more or less stringent. For example a relation "is the same type of object" may manifest the equivalence class set composed of vehicle objects, cyclist objects, static objects, or it may manifest the equivalence class set composed of aggressive car's driver objects, AV objects, absent-minded car's driver objects [20], [21], electric bicycle's driver objects, and so on.

Let us define the states \& attributes of the equivalence class of $x$ set $A^{[x]}$ as the set of the entail dynamic motion information (like moving forward, being still, turning right) and the information indicating an immediate action that is taking place while the snapshot in time is being taken (like indicator activated and honking, raining). Let $\mathcal{P}\left(A^{[x]}\right)$ be the power set of $A^{[x]}$ and $A:=\bigcup_{[x] \in V} \mathcal{P}\left(A^{[x]}\right)$ the states \& attributes set, then we define the states \& attributes function as follows:

$$
f_{\text {sa }}: \mathcal{V} \rightarrow A, \quad x \mapsto f_{\text {sa }}(x)
$$

where each $x \in \mathcal{V}$ is associated to an element of $A$, i.e. to a subset of states \& attributes of the equivalence class of $x$ set, Fig.5. Similarly we define the states \& attributes from $x$ 's point of view function, or $x$-states \& attributes function as follows:

$$
f_{s a}^{x}: \mathcal{V}_{x} \rightarrow A_{x}, \quad y \mapsto f_{s a}^{x}(y),
$$

where $A_{x}:=\bigcup_{[y] \in V_{x}} \mathcal{P}\left(A_{x}^{[y]}\right), A_{x}^{[y]}$ is the states \& attributes of the equivalence class of $y$ from $x$ 's point of view set (or $x$-states \& attributes of the equivalence class of $y$ set) and $f_{s a}^{x}(y)$ is the states \& attributes from $x$ 's point of view set, or $x$-states \& attributes set, i.e. each $y \in \mathcal{V}_{x}$ is associated to a subset of $A_{x}$.

Remark 3. Let $x$ be an observer, then the set $A_{x}$, unlike the set $A$, may not completely characterise the objects in $\mathcal{V}_{x}$. In fact, $x$ may not be able (not having all the capabilities) to attribute all the states and attributes necessary to characterise the objects it sees/perceives. For example, it may not be able to identify wind speed and the amount of precipitation.

Example 5. States \& attributes set

Let us suppose the states \& attributes of the equivalence class of $x$ set, $A^{[x]}$, where $[x]$ is the equivalence class of vehicle, is made up of the elements $\{i, f, s, r, l, h\}$ (where $i=$ indicator activated, $f=$ moving forward, s=being still, $r=$ turning right, $l=$ turning left, $h=$ honking). Then in the example shown in Fig.la we could assign the states \& attributes set $f_{s a}(x)=\{f\}$ to the car $x$. If the tree hadn't been in the scenery and the car driver had seen the bicycle it could sound the horn, and then the states \& attributes set could take the form $f_{s a}(x)=\{f, h\}$. In this case if the cyclist $(y)$ is deaf, for instance, it would attribute the set $f_{\text {sa }}^{y}(x)=\{f\}$.

Example 6. States \& attributes set, Environmental Conditions.

Let us consider now the Environmental Conditions of the ODD (Operational Design Domain) classification, [22], which are divided into four subcategories: weather, illumination, particulate matter, and road weather. All these subcategories are objects of the set $\mathcal{V}^{s}$. As such each of these subcategories have a position, given by the function $\mathcal{C}$. This position can be a region of $\mathcal{S}$, or even the whole portion of space $\mathcal{S}$. In addition to the position, we can also assign the states \& attributes function and set. For instance, the states \& attributes of the equivalence class of $x$ set $A^{[x]}$ for the object weather can be composed by the elements rain, temperature, wind, and snow, which can be characterised by some adjectives, like low, moderate and heavy. Then the object weather can be associated to one or more of the elements $\{$ rain low, rain moderate, rain heavy,$\ldots$, snow heavy $\}=A^{[x]}$ by the states \& attributes function.

At this point we have all the necessary ingredients to define a scene. 
Definition 1 (Scene). A scene $\mathcal{E}_{x}(\mathcal{S}, t)$ of the portion of space $\mathcal{S}$ at time $t$ from $x$ 's point of view is defined as the 3-tuples $\mathcal{E}_{x}(\mathcal{S}, t):=\left(\mathcal{V}_{x}, \mathcal{C}_{x}\left(\mathcal{V}_{x}\right), f_{s a}^{x}\left(\mathcal{V}_{x}\right)\right)$ where $\mathcal{V}_{x}$ is the object set from x's point of view at time $t, \mathcal{C}_{x}\left(\mathcal{V}_{x}\right)$ and $f_{s a}^{x}\left(\mathcal{V}_{x}\right)$ are the $x$-position set and $x$-states \& attributes set respectively.

Remark 4. If we are in a simulated world where "a scene can be complete and uncertainty-free as from an omniscient observer's point of view", [1, p. 983], then x's point of view sets have to be replaced by omniscient observer's point of view sets by obtaining the complete scene $\mathcal{E}(\mathcal{S}, t)=\left(\mathcal{V}, \mathcal{C}(\mathcal{V}), f_{\text {sa }}(\mathcal{V})\right)$ where $\mathcal{V}$ is the object set at time $t, \mathcal{C}(\mathcal{V})$ and $f_{\text {sa }}(\mathcal{V})$ are the position set and states \& attributes set respectively.

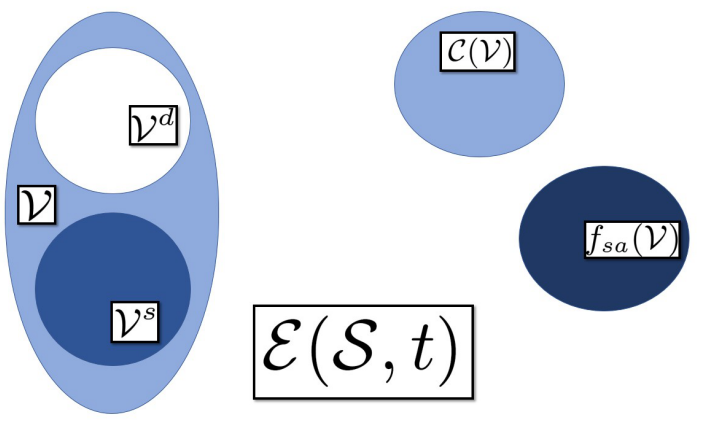

Fig. 6: Scene sets.

If we restrict the definition of scene to the set of static objects, instead of the set of all static and dynamic objects, we get the scenery. Let $\mathcal{V}_{x}^{s}$ be the static object set from $x$ 's point of view, i.e. $\mathcal{V}_{x}^{s}:=\mathcal{V}^{s} \cap \mathcal{V}_{x}$, then we can define the scenery as follows:

Definition 2 (Scenery). A scenery $\mathcal{Y}_{x}(\mathcal{S})$ of the portion of space $\mathcal{S}$ is defined as the 3-tuples $\mathcal{Y}_{x}(\mathcal{S}):=$ $\left(\mathcal{V}_{x}^{s}, \mathcal{C}_{x}\left(\mathcal{V}_{x}^{s}\right), f_{s a}^{x}\left(\mathcal{V}_{x}^{s}\right)\right)$ where $\mathcal{V}_{x}^{s}$ is the set of the $x$-static object, $\mathcal{C}_{x}\left(\mathcal{V}_{x}^{s}\right)$ and $f_{s a}^{x}\left(\mathcal{V}_{x}^{s}\right)$ are the $x$-static position set (the $x$-position set of the set of the $x$-static object) and $x$-static states \& attributes set (the states \& attributes set of the set of the $x$-static object) respectively.

Remark 5. Similarly to the definition of a scene, even for the definition of scenery we can consider the viewpoint of the omniscient observer, and therefore the complete and uncertainty-free scenery is defined by replacing the x-point of view sets by omniscient observer's point of view sets: $\mathcal{Y}(\mathcal{S}):=\left(\mathcal{V}_{s}, \mathcal{C}\left(\mathcal{V}_{s}\right), f_{s a}\left(\mathcal{V}_{s}\right)\right)$ where $\mathcal{V}_{s}$ is the set of the static object, $\mathcal{C}\left(\mathcal{V}_{s}\right)$ and $f_{s a}\left(\mathcal{V}_{s}\right)$ are the static position set (the position set of the set of the static object) and static states $\&$ attributes set (the states \& attributes set of the set of the static object) respectively.

In this way, we have the scenery implicitly included in the scene definition.

\section{DEFINING THE TERM SITUATION}

In order to define the term situation we introduce the goals $\&$ values position functions.

Let us firstly define the goals \& values position from x's point of view function, or $x$-goals \& values position function, as the function

$$
\overline{\mathcal{C}}_{x}: \mathcal{V}_{x} \rightarrow \mathcal{S}, \quad y \mapsto \overline{\mathcal{C}}_{x}(y) .
$$

which associate each object $y \in \mathcal{V}_{x}$ to the position of it in the near future, based on $x$ 's expectation (see Fig.7a).

Remark 6. The position of $y \in \mathcal{V}_{x}$ in the near future, based on x's expectation, will depend not only on y's goal, but also on the values that $x$ attributes to y (see Example 8).

Example 7. Goals \& values positions

Let us consider the example illustrated in Example 1 and the object set from car driver's $(x)$ point of view, $\mathcal{V}_{x}$, represented in Fig.3a.

Because $x$ is not able to see the bicycle $y$ behind the tree, it cannot predict a position for $y$ in the near future: in the future it sees itself crossing the intersection without braking. In reality, however, the bicycle exists. What happens in the future is an accident, as shown in Fig.7b.

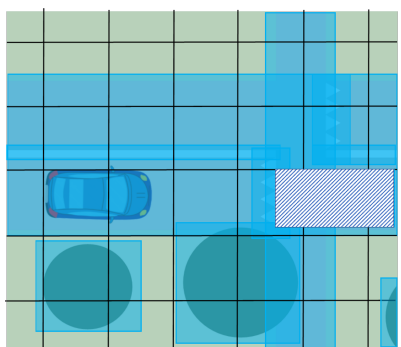

(a) Goals \& values position from the $x$ (car driver) point of view set, $\overline{\mathcal{C}}_{x}\left(\mathcal{V}_{x}\right)$. In the car driver forecast all the objects present in the portion of space $\mathcal{S}$ will have the same position also in the future (blue solid fill) except itself: the car driver imagines itself (striped fill) having passed the intersection with the cycle lane.

Fig. 7: $x$-goals \& values position set and goals \& values position set.

\section{Example 8. Goals \& values positions}

If a car driver $y$ does not turn on the indicator, then our observer $x$ will expect that the car will go straight in the near future. But let us suppose that $x$ gives $y$ the value of being a unruly driver, then $x$ might expect $y$ to turn right or turn left equally.

Example 9. Goals \& values positions (change over time) Now let us consider the car driver $y$ does not turn the indicator on at time $t$ and our subject $x$ expects the car to go straight in the near future. However, it could happen the car driver y turns right/left at time $t+\Delta t$. It is even possible that our subject $x$, some seconds later, at time $t+\Delta t / 2$, could expect the car driver y to turn somewhere because it realises the car driver $y$ decelerates. Then, the image set of $\mathcal{V}_{x}$ under the $x$-goals \& values position function can change over time.

The set $\overline{\mathcal{C}}_{x}\left(\mathcal{V}_{x}\right)$ is called goals \& values position from $x$ 's point of view set, or $x$-goals \& values position set for brevity. In general we expect that $\overline{\mathcal{C}}_{x}(y)=\mathcal{C}_{x}(y)$ if $y \in \mathcal{V}_{x}^{s}$, even if this is not always true; a tree, which belongs to the set $\mathcal{V}^{s}$, can break and fall, for instance. 
Let us consider the couple $(\mathcal{V}(\mathcal{S}, t), \mathcal{C}(\mathcal{V}(\mathcal{S}, t)))$. If we want to predict the future of this couple, as real snapshot, we could think of considering the couple $(\mathcal{V}(\mathcal{S}, t+\Delta t), \mathcal{C}(\mathcal{V}(\mathcal{S}, t+\Delta t)))$. But, this couple just predicts the future at an exact time, it is not the real goal of the objects in $\mathcal{V}(\mathcal{S}, t)$ and moreover the set $\mathcal{V}(\mathcal{S}, t)$ could be different from $\mathcal{V}(\mathcal{S}, t+\Delta t)$.

For this purpose we define the goals \& values position function from a real point of view as follows:

$$
\overline{\mathcal{C}}: \mathcal{V} \rightarrow \mathcal{S}, \quad y \mapsto \overline{\mathcal{C}}(y)
$$

where the goals \& values position function associates each object $y$ in $\mathcal{V}$ to the dimensional coordinate of $y$ in the near future, based on $y$ 's expectation, as illustrated in Fig. $7 \mathrm{~b}$ and Example 7. The goals \& values position function knows the goals of each object at time $t$, i.e. what each object in $\mathcal{V}$ is going to do. In order to nursue the definitions given in 511 we

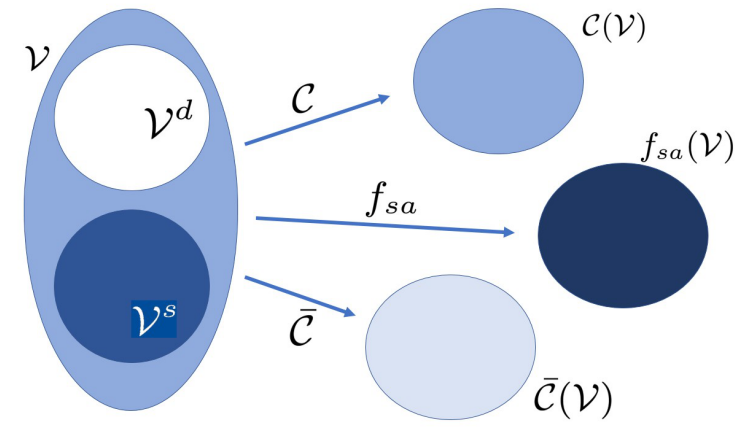

Fig. 8: Sets and functions diagram. From object set we defined three functions: the position function $\mathcal{C}$, the states \& attributions function $f_{s a}$ and the goals \& values position function $\overline{\mathcal{C}}$. Here we illustrate the domain of the three functions, the object set, and the image sets of the functions.

need to introduce a relevant function which filters the relevant information from a given set of the whole information.

Let us firstly define the enlarged situation $\operatorname{set}, \mathcal{N}_{x}^{e}(\mathcal{S}, t)$ (or $\mathcal{N}_{x}^{e}$ if no ambiguity concurring), as the 4-tuples made up of the $x$-object, $x$-position, $x$-states $\&$ attributions and $x$-goals $\&$ values position sets $\mathcal{N}_{x}^{e}:=\left(\mathcal{V}_{x}, \mathcal{C}_{x}\left(\mathcal{V}_{x}\right), f_{s a}^{x}\left(\mathcal{V}_{x}\right), \overline{\mathcal{C}}_{x}\left(\mathcal{V}_{x}\right)\right)$.

The relevant function operates on the enlarged situation set as a compositions of two functions: firstly the relevant object function filters out all the irrelevant objects from $\mathcal{N}_{x}^{e}$ and all the information about them; secondly, on the remaining sets, the relevant information function filters out all the irrelevant information which are not related with the presence or absence of the objects.

We define a $x$-relevant function any function defined as follows:

Definition 3. Relevant function, $f_{r}^{x}$ Let $\mathcal{N}_{x}^{e}:=\left(\mathcal{V}_{x}, \mathcal{C}_{x}\left(\mathcal{V}_{x}\right), f_{s a}^{x}\left(\mathcal{V}_{x}\right), \overline{\mathcal{C}}_{x}\left(\mathcal{V}_{x}\right)\right)$ be the enlarged situation of the $x$-object set $\mathcal{V}_{x}$. A relevant function $f_{r}^{x}$ is defined as each function which can be rewritten as the composition of two functions which operate as follows:

$$
f_{r}^{x}: \mathcal{N}_{x}^{e} \stackrel{f_{r o}^{x}}{\longrightarrow} \mathcal{N}_{x o}^{e} \stackrel{f_{r i}^{x}}{\longrightarrow} \mathcal{N}_{x}
$$

where $\mathcal{N}_{x o}^{e}:=\left(\mathcal{V}_{x}^{r}, \mathcal{C}_{x}\left(\mathcal{V}_{x}^{r}\right), f_{s a}^{x}\left(\mathcal{V}_{x}^{r}\right), \overline{\mathcal{C}}_{x}\left(\mathcal{V}_{x}^{r}\right)\right)$, with $\mathcal{V}_{x}^{r}$ any subset of $\mathcal{V}_{x}$; and $\mathcal{N}_{x}:=\left(\mathcal{V}_{x}^{r}, \mathcal{C}_{x}^{r}\left(\mathcal{V}_{x}^{r}\right), f_{s a}^{x r}\left(\mathcal{V}_{x}^{r}\right), \overline{\mathcal{C}}_{x}^{r}\left(\mathcal{V}_{x}^{r}\right)\right)$, with
$\mathcal{C}_{x}^{r}\left(\mathcal{V}_{x}^{r}\right), f_{s a}^{x r}\left(\mathcal{V}_{x}^{r}\right)$ and $\overline{\mathcal{C}}_{x}^{r}\left(\mathcal{V}_{x}^{r}\right)$ any subset of $\mathcal{C}_{x}\left(\mathcal{V}_{x}^{r}\right), f_{s a}^{x}\left(\mathcal{V}_{x}^{r}\right)$ and $\overline{\mathcal{C}}_{x}\left(\mathcal{V}_{x}^{r}\right)$ respectively.

The set $\mathcal{V}_{x}^{r}$ is called a $x$-relevant object set and the sets $\mathcal{C}_{x}^{r}\left(\mathcal{V}_{x}^{r}\right) f_{s a}^{x r}\left(\mathcal{V}_{x}^{r}\right)$ and $\overline{\mathcal{C}}_{x}^{r}\left(\mathcal{V}_{x}^{r}\right)$ are $x$-relevant position, $x$ relevant states \& attributions and $x$-relevant goals \& values position sets respectively. At this point we have all the necessary ingredients to define a situation.

Definition 4 (Situation). A situation $\mathcal{N}_{x}(\mathcal{S}, t)$ of the portion of space $\mathcal{S}$ at time $t$ from $x$ 's point of view is defined as any 4-tuples $\mathcal{N}_{x}(\mathcal{S}, t):=\left(\mathcal{V}_{x}^{r}, \mathcal{C}_{x}^{r}\left(\mathcal{V}_{x}^{r}\right), f_{s a}^{x r}\left(\mathcal{V}_{x}^{r}\right), \overline{\mathcal{C}}_{x}^{r}\left(\mathcal{V}_{x}^{r}\right)\right)$ where $\mathcal{V}_{x}^{r}$ is a x-relevant object set, $\mathcal{C}_{x}^{r}\left(\mathcal{V}_{x}^{r}\right), f_{s a}^{x r}\left(\mathcal{V}_{x}^{r}\right)$ and $\overline{\mathcal{C}}_{x}^{r}\left(\mathcal{V}_{x}^{r}\right)$ are $x$-relevant position, $x$-relevant states \& attributions and $x$-relevant goals \& values position sets respectively.

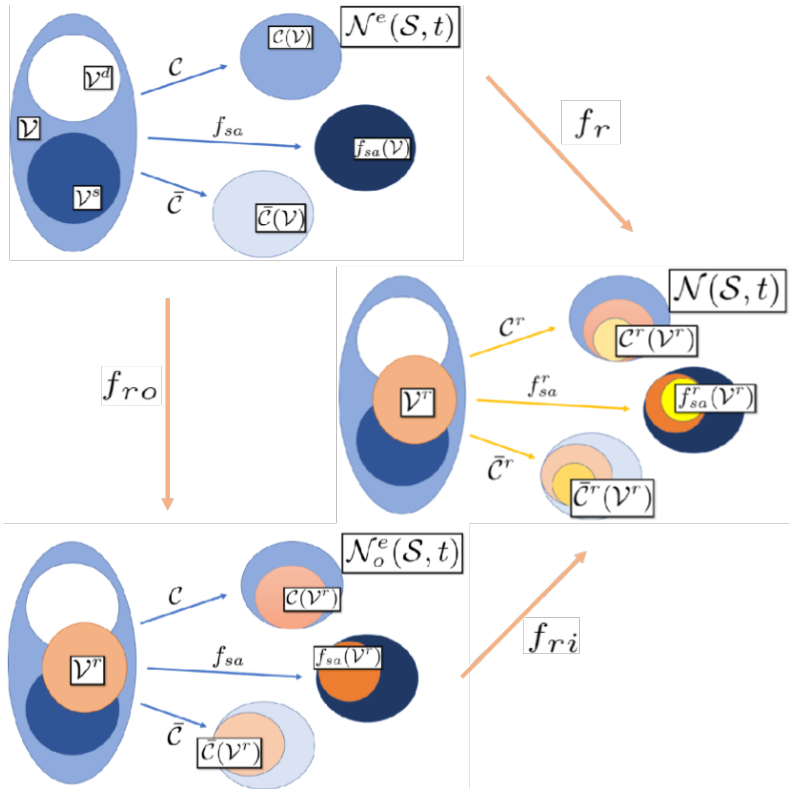

Fig. 9: Relevant function, $f_{r}$, diagram from the enlarged situation $\mathcal{N}^{e}$ to the situation $\mathcal{N}$.

Remark 7. From an omniscient observer the situation become complete and uncertainty-free, then it is defined by replacing the $x$-point of view sets and functions by omniscient observer's point of view sets and functions.

Given the relevant function $f_{r}$, Fig.9, defined as follows:

$$
f_{r}: \mathcal{N}^{e} \stackrel{f_{\text {ro }}}{\longrightarrow} \mathcal{N}_{o}^{e} \stackrel{f_{\text {ri }}}{\longrightarrow} \mathcal{N}
$$

where $\mathcal{N}^{e}:=\left(\mathcal{V}, \mathcal{C}(\mathcal{V}), f_{\text {sa }}(\mathcal{V}), \overline{\mathcal{C}}(\mathcal{V})\right)$ is the enlarged situation, $\mathcal{N}_{o}^{e}:=\left(\mathcal{V}^{r}, \mathcal{C}\left(\mathcal{V}^{r}\right), f_{s a}\left(\mathcal{V}^{r}\right), \overline{\mathcal{C}}\left(\mathcal{V}^{r}\right)\right)$, with $\mathcal{V}^{r}$ any subset of $\mathcal{V}$; and $\mathcal{N}:=\left(\mathcal{V}^{r}, \mathcal{C}^{r}\left(\mathcal{V}^{r}\right), f_{\text {sa }}^{r}\left(\mathcal{V}^{r}\right), \overline{\mathcal{C}}^{r}\left(\mathcal{V}^{r}\right)\right)$, with $\mathcal{C}^{r}\left(\mathcal{V}^{r}\right), f_{s a}^{r}\left(\mathcal{V}^{r}\right)$ and $\overline{\mathcal{C}}^{r}\left(\mathcal{V}^{r}\right)$ any subset of $\mathcal{C}\left(\mathcal{V}^{r}\right), f_{s a}\left(\mathcal{V}^{r}\right)$ and $\overline{\mathcal{C}}\left(\mathcal{V}^{r}\right)$ respectively, a situation $\mathcal{N}(\mathcal{S}, t)$, or simply $\mathcal{N}$, of the portion of space $\mathcal{S}$ at time $t$ is defined as the 4-tuples $\mathcal{N}(\mathcal{S}, t):=\left(\mathcal{V}^{r}, \mathcal{C}^{r}\left(\mathcal{V}^{r}\right), f_{s a}^{r}\left(\mathcal{V}^{r}\right), \overline{\mathcal{C}}^{r}\left(\mathcal{V}^{r}\right)\right)$ where $\mathcal{V}^{r}$ is a relevant object set, $\mathcal{C}^{r}\left(\mathcal{V}^{r}\right), f_{\text {sa }}^{r}\left(\mathcal{V}^{r}\right)$ and $\overline{\mathcal{C}}^{r}\left(\mathcal{V}^{r}\right)$ are relevant position, relevant states \& attributions and relevant goals \& values position sets respectively.

\section{DEFINING THE TERM SCENARIO}

In order to define a scenario we have to define goals \& values and actions \& events functions first, Fig.11. 
Definition 5 (Goals \& values function). A goals \& values function is the function that describes the path that the object $y$ takes in order to reach its goal, i.e. to reach the position $\overline{\mathcal{C}}(y)$

$$
f_{g v}: \mathcal{C}(\mathcal{V}) \rightarrow \overline{\mathcal{C}}(\mathcal{V}) \times T, \quad \mathcal{C}(y) \mapsto(\overline{\mathcal{C}}(y), \bar{t}) .
$$

Because the function $\mathcal{C}$ depends on time $t$ as well as the dimensional coordinate of $y$, the function $f_{g v}$ is able to compute the goal-time $\bar{t}$, the time in which the object y reaches the position $\overline{\mathcal{C}}(y)$.

Remark 8. The goals \& values function is updated at each time step, and its form depends on the interactions of the object with the environment. Let us suppose that the ego vehicle $y$ is in a car following condition at time $t_{1}$. At time $t_{1}$ the function $f_{\text {gv }}$ will be given by $f_{g v}\left(\mathcal{C}\left(y\left(t_{1}\right)\right)\right)=\left(\mathcal{C}\left(y\left(t_{1}\right)\right)+v_{y} \bar{t}, \bar{t}\right)$, where $v_{y}$ is the constant speed of the vehicle $y$. Let us suppose now that the lead vehicle brakes suddenly at time $t_{2}$. Then, after a reasonable reaction time $\tau$ (between about 0.7 to 2 seconds for HOVs [23], and about 0.5 for AVs [24]), vehicle y will also start to brake. Therefore, at time $t_{2}+\tau$ the function $f_{g v}(\mathcal{C}(y))$ will change its form or the value of the parameters. It could decrease the speed $v_{y}$ or it could involve a deceleration which in the car following was not relevant. Furthermore, the form/values of the parameters may be also different for $A V$ compared to $\mathrm{HOV}$, due to their different reaction times.

Definition 6 (Actions \& events function). A actions \& events function is the function that describes the path that the object $y$, located in $\mathcal{C}(y(t))$ at time $t$, takes in order to reach its position at time $t+\Delta t$, i.e. to reach the position $\mathcal{C}(y(t+\Delta t))$

$$
\begin{aligned}
f_{a e}: \mathcal{C}(\mathcal{V}(\mathcal{S}, t)) \times T & \rightarrow \mathcal{C}(\mathcal{V}(\mathcal{S}, t+\Delta t)) \\
(\mathcal{C}(y(t)), t+\Delta t) & \mapsto \mathcal{C}(y(t+\Delta t)) .
\end{aligned}
$$

Remark 9. In general it is not true that $\overline{\mathcal{C}}(y(t))=\mathcal{C}(y(\bar{t}))$. Indeed, the object y could change its goals over the time while $\mathcal{C}(y(\bar{t}))$ represents the real position it reaches at time $\bar{t}$.

At this point we define the goals \& values from $x$ 's point of view function, or $x$-goals \& values function, as follows:

Definition 7 ( $x$-goals \& values function). A x-goals \& values function is the function that describes the path that the object $y$ takes in order to reach the position $\overline{\mathcal{C}}_{x}(y)$ from $x$ 's point of view

$$
f_{g v}^{x}: \mathcal{C}_{x}\left(\mathcal{V}_{x}\right) \rightarrow \overline{\mathcal{C}}_{x}\left(\mathcal{V}_{x}\right) \times T, \quad \mathcal{C}_{x}(y) \mapsto\left(\overline{\mathcal{C}}_{x}(y), \bar{t}_{x}\right)
$$

where $\bar{t}_{x}$ is the time the object $y$ reaches the position $\overline{\mathcal{C}}_{x}(y)$ based on $x$ 's expectation.

Definition 8 ( $x$-actions \& events function). A x-actions \& events function is the function that describes the path that the object $y$, located in $\mathcal{C}_{x}(y(t))$ at time $t$, takes in order to reach its position at time $t+\Delta t$, i.e. to reach the position $\mathcal{C}_{x}(y(t+\Delta t))$ from $x$ 's point of view

$$
\begin{aligned}
f_{a e}^{x}: \mathcal{C}_{x}\left(\mathcal{V}_{x}\right) \times T & \rightarrow \mathcal{C}_{x}\left(\mathcal{V}_{x}\right) \\
\left(\mathcal{C}_{x}(y(t)), t+\Delta t\right) & \mapsto \mathcal{C}_{x}(y(t+\Delta t)) .
\end{aligned}
$$

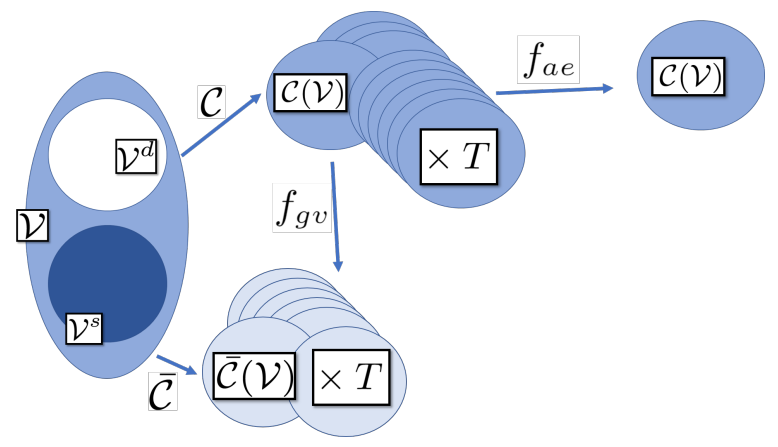

Fig. 10: Goals \& values and actions \& events functions

The function $f_{g v}^{x}$ takes the name of $x$-goals \& values function because intrinsically contains the information of the values of the object $y$ (from $x$ 's point of view).

\section{Example 10. $x$-goals \& values function}

In order to make more explicit how x-goals \& values function can represent the values of the object $y$ let us illustrate an example. Let us suppose the observer $x$ considers $y_{1}$ an aggressive driver and $y_{2}$ a distracted driver, then the function $f_{g v}^{x}\left(\mathcal{C}\left(y_{1}\right)\right)$ should take a form which is different from $f_{g v}^{x}\left(\mathcal{C}\left(y_{2}\right)\right)$. A possible choice to include these information in the model is to describe the function $f_{g v}^{x}$ as a piecewise function depending on the object $y$ and the values that the observer $x$ decides to give to $y$. Let us denote, for instance, $\left[y_{1}\right],\left[y_{2}\right],\left[y_{3}\right]$ be the set of the aggressive driver, distracted driver and driverless respectively, then we can define the function $f_{g v}^{x}$ in the following way

$$
f_{g v}^{x}(\mathcal{C}(y))= \begin{cases}g_{1}(y) & \text { if } x, y \in\left[y_{1}\right] \\ g_{2}(y) & \text { if } x \in\left[y_{1}\right], y \in\left[y_{2}\right] \\ g_{3}(y) & \text { if } x \in\left[y_{1}\right], y \in\left[y_{3}\right] \\ g_{4}(y) & \text { if } x \in\left[y_{3}\right], y \in\left[y_{1}\right] \\ g_{5}(y) & \text { if } x \in\left[y_{3}\right], y \in\left[y_{2}\right] \\ g_{6}(y) & \text { if } x, y \in\left[y_{3}\right] \\ g_{7}(y) & \text { if } x \in\left[y_{2}\right]\end{cases}
$$

where if $x \in\left[y_{2}\right]$ (a distracted observer) it is not able to distinguish the different modes of the objects it sees (otherwise we could introduce a stochastic parameter in order to define an almost distracted driver). Now let us suppose $z$ is an honking driver, then we can expect it affects the guide of the distracted driver and the perception of $x$. We can include the information in the function by substituting the functions $g_{2}, g_{5}$ and $g_{7}$ with $g_{2}\left(y \mid z \in \mathcal{V}_{x}\right), g_{5}\left(y \mid z \in \mathcal{V}_{x}\right)$ and $g_{7}\left(y \mid z \in \mathcal{V}_{x}\right)$ respectively.

When the $x$-goals \& values of object $y$ and the goals \& values of $y$ does not overlap, or deviate too much, then the probability of conflict may increase. In this way the number of conflicts or safety critical events (SCEs) can be counted and followed-up in the simulation.

Let us now define a scenario as follows.

Definition 9 (Scenario). A scenario $\mathcal{O}_{\mathcal{T}}^{x}(\mathcal{S})$ on the portion of space $\mathcal{S}$ in the time set $\mathcal{T}$ from $x$ 's point of view is defined as the $\mathcal{O}_{\mathcal{T}}^{x}(\mathcal{S}):=\left(\mathcal{E}_{t}^{x}(\mathcal{S}), f_{g v}^{x}, f_{a e}^{x}\right)_{t \in \mathcal{T}}$, where the time set is a set of ordered sequence of times $\mathcal{T}=\left\{t_{i}: t_{i}<t_{i+1}, i=\right.$ $0, \ldots, n-1\}$. 


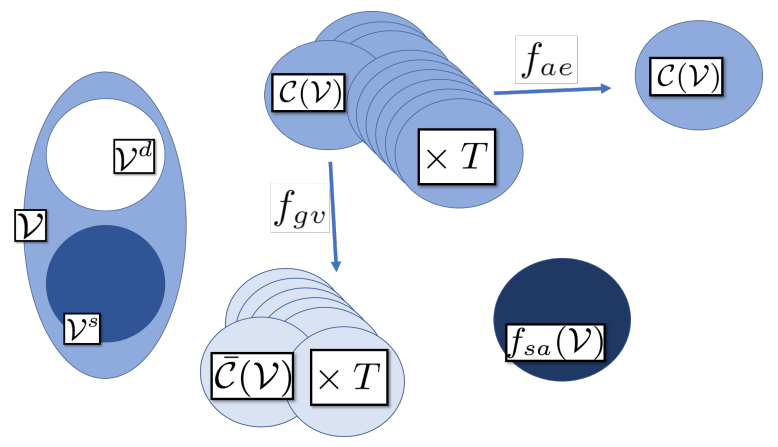

Fig. 11: Scenario diagram

Remark 10. Differently from scene and situation definitions, which have been defined through set tuples, in the case of scenario definition it is necessary to know the functions $f_{g v}^{x}, f_{a e}^{x}$ which describe how the evolution of scene happens.

\section{SCENARIO FROM REAL DRIVING DATA}

In order to show the framework capability of representing realistic and rare-conditions, let us consider two public repositories of real driving data: UAH-DriveSet [25], and DR(eye)VE [26]. In the first one the data are recorded by using the smartphone application DriveSafe and the sensors on the smartphone (inertial sensors, GPS, camera and internet access) are used to $\log$ and recognize driving maneuvers and infer behaviors from them. In the second one, the data are acquired both from the driver gaze through an eye tracking device, and from a roof-mounted camera, in order to have both the driver's and the vehicle's point of view, and to predict the driver's focus of attention. The main aim of this section is to consider some scenarios described in these datasets and show how these scenarios can be rewritten according to our definitions.

1) Object and states \& attributes sets: In TABLE I, the objects and their respective states \& attributes are shown. We observe that the attributes can belong to different classes: in the UAH-DriveSet the ego-object has attributes related to its behavior, while in the DR(eye)VE to the attention it shows to the various events. However, our definition allows us to consider more classes simultaneously.

2) Position set: Both datasets provide precise positions of the ego-vehicle through geographic coordinates, speed at any time step, the course and accelerations along the axes of the ego-vehicle. The first information (geographic coordinates) is an element of the position set, precisely the position of the egovehicle object: in both datasets the position is given through the latitude and longitude coordinates. In addition to those coordinates, the first dataset also has information on altitude.

3) $f_{a e}$ and $f_{g v}$ functions: The other three information sets (speed, course and acceleration) are the parameters that define the functions $f_{a e}$ and $f_{g v}$. The acceleration, in the first dataset, also takes into account the acceleration along the vertical axis, and this aspect, together with the altitude information, makes the functions $f_{a e}$ and $f_{g v}$ able to describe the movements of the ego-vehicle in going up and down from hills. Due to this piece of information, we can consider the perceptions of the ego-speed when going up and down from a hill through the function $f_{g v}$, [27]. In Fig.(12) we show how the speed of the

\begin{tabular}{c|c|c} 
Object & UAH-DriveSet & DR(eye)VE \\
\hline Ego-object & Drowsy, Normal, Aggressive & Inattentive, Attentive \\
\hline Light & & Morning, Evening, Night \\
\hline Weather & & Sunny, Cloudy, Rainy \\
\hline Landscape & & $\begin{array}{c}\text { Downtown, Countryside, } \\
\text { Highway }\end{array}$ \\
\hline Road & Secondary, Motorway & \\
\hline Traffic & Number of vehicles & \\
\hline Lane & Number of lanes & \\
\hline Max speed & Number $(\mathrm{km} / \mathrm{h})$ &
\end{tabular}

TABLE I: States \& attributes set

vehicle D4 (UAH-DriveSet) varies as the altitude changes. The elevation parameter can be added to SUMO in several ways [28], but the effects on driving are not taken into account yet, such as the perception of speed.
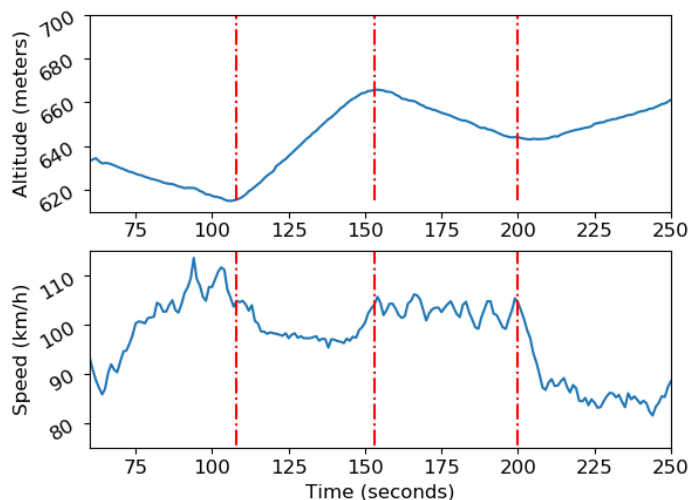

Fig. 12: Altitude (top) and relative driver D4's speed (bottom).

Another aspect of the driving behaviour that we extract from real driving data, and we can describe through our definition (object and position sets) is the centering of the vehicles with respect to the center of the lane. In Fig. 13 (top), two vehicles (D1 and D2 of the UAH-DriveSet) trajectories with respect to the center of the lane are traced. Both the vehicles show fluctuations in lane-keeping but these deviations have different meaning: one is intentional (i.e. overtaking or intent to overtake) and the other is unintentional (i.e. due to an impairment or distraction as well as they could be related to drifting). In fact, we observe that on average D1 drives on the right side of its lane (the gray dashed line), while D2 is centered (black dashed line) and the fluctuations' amplitude is greater and more irregular, as if D2 aims to overtake a vehicle ahead. Moreover, we note in Fig.13 (bottom) that, unlike D1, D2 has a lead vehicle during the whole scenario and therefore its fluctuations are due to the intention of overtaking. At time $70 \mathrm{~s}, \mathrm{D} 2$ tries to overtake the lead vehicle and it identifies as a new lead vehicle at $120 \mathrm{~m}$ away, i.e. the oncoming traffic in the opposite lane. Unlike D2, D1 does not overtake anyone, it keeps to the right, and therefore its fluctuations are unintentional. In the supplementary material [29] we have simulated in detail a one-minute complex scenario that features the same settings as this observed sequence from UAHDriveSet.

\section{DISCUSSION}

By comparing our definitions of scene/scenario with, for example, the scene/scenario build with the open source 

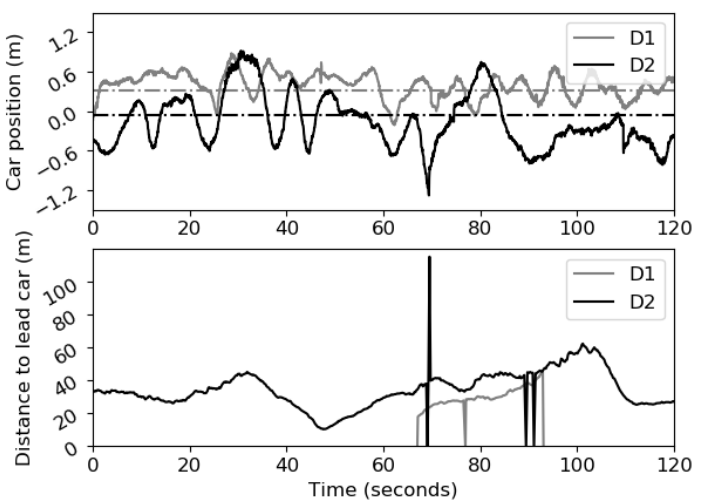

Fig. 13: Comparison of two driving behaviours on secondary roads. Dashed lines show the driver behaviour on average.

SUMO software, we note that in several aspects there is a natural overlap. Each object in SUMO belongs to a class of equivalence called type: each vehicle, according to SUMO, can be a passenger, bicycle, pedestrian, truck, tram or rail, as well as each edge can be a highway.bridleway, highway.bus_guideway, highway.cycleway or highway.pedestrian. Moreover, according to the equivalence class to which the object belongs, it is characterised by specific attributes, exactly as the $f_{s a}$, and by a position, which can be changeable over time or not.

SUMO also includes the 'agents' more or less in 'reactive' mode, the agents/objects are not generally 'interactive'. The driver behavior is simulated in compliance with the ACME-Driver Model [12], i.e. the model is able to produce elementary forms of strategic-level behavior, such as individual routing capability or the ability of drivers to recognize high congestion and use alternative routes, but a more comprehensive perception mechanism is not yet implemented. This is a limitation in creating situations where the human/machine perception and relative point of views or 'model in-compliant info' cannot be represented. In our definitions, we actually can represent interactive agent behavior, relative perception capacity and similar advanced dynamic features of road-user behavior.

\section{CONCLUSIONS}

The approach presented in this work gives a mathematical form to the definitions given in [1], which makes it possible to consider the point of view of each object present in the scenario and to treat it as an ego-object (ego-HOV, ego-AV and ego -VRU), i.e. a subject of the scenario. As previous studies shown, the driver's choices (to let the pedestrians cross the streets, overtake the bicycle, sound the horn, etc.) and the driving style change according to the country [30], [31], the topology of the road [32], [33], the type, the condition as well as the perception of the driver [34], [35].

Through our definitions, the ego-object can be implemented taking into account not only its characteristics (i.e. whether it is aggressive, impulsive, cooperative or strategic, autonomous, automated or human-operated [36], [37], [38], [39], [40]), but also what it is able to see and perceive, its cognitive aspects, and what it expects the other objects present in the scenario to behave (which does not always correspond to the truth) so that the ego-object can react accordingly. In this paper, we have taken advantage of the flexibility of the set theory to design a framework that may be adapted to any software and in any future research development. In the near future, we will use this new approach to build complex scenarios composed of dynamic objects with their subjective point of views. Moreover, these dynamic objects will perform manoeuvres taking into account their point of views and the manoeuvres of the other dynamic objects placed in the scenario, as if they were pieces of a larger and more complex puzzle. The point of views of each of the objects within the big scenario can be shared with other objects (e.g., in the context of V2x) leading to a collective cooperation between communicating objects. Consequently the decisions of the objects (to deviate path, overtake, etc.) may depend not only on what happens within the scenario, but also on these communications and thus on what happens in the macroscopic scenario description.

\section{ACKNOWLEDGMENT}

This work is financially supported by the Swedish Innovation Agency Vinnova for grant 2018-02891 and Chalmers Area of Advance Transport for DS-Auto.

\section{REFERENCES}

[1] S. Ulbrich, T. Menzel, A. Reschka, F. Schuldt, and M. Maurer, "Defining and Substantiating the Terms Scene, Situation, and Scenario for Automated Driving," in 2015 IEEE 18th International Conference on Intelligent Transportation Systems. IEEE, Sep. 2015, pp. 982-988.

[2] R. Queiroz, T. Berger, and K. Czarnecki, "GeoScenario: An Open DSL for Autonomous Driving Scenario Representation,” in 2019 IEEE Intelligent Vehicles Symposium (IV). IEEE, Jun. 2019, pp. 287-294.

[3] R. Studer, R. Benjamins, and D. Fensel, "Knowledge engineering: Principles and methods," Data Knowl. Eng., vol. 25, pp. 161-197, 1998.

[4] N. Guarino, D. Oberle, and S. Staab, "What is an ontology?" in Handbook on Ontologies, S. Staab and R. Studer, Eds. Springer, 2009.

[5] G. Bagschik, T. Menzel, and M. Maurer, "Ontology based scene creation for the development of automated vehicles," 2018 IEEE Intelligent Vehicles Symposium (IV), pp. 1813-1820, 2017.

[6] T. Menzel, G. Bagschik, L. Isensee, A. Schomburg, and M. Maurer, "Detaillierung einer stichwortbasierten szenariobeschreibung für die durchführung in der simulation am beispiel von szenarien auf deutschen autobahnen," in Workshop Fahrerassistenzsysteme und automatisiertes Fahren, Walting im Altmühltal: Uni-DAS e.V., vol. 12, 2018, pp. 15-26.

[7] F. Schuldt, F. Saust, B. Lichte, M. Maurer, and S. D. Scholz, "Effiziente systematische testgenerierung für fahrerassistenzsysteme in virtuellen umgebungen," in AAET - Automatisierungssysteme, Assistenzsysteme und eingebettete Systeme für Transportmitte, 2013.

[8] T. Menzel, G. Bagschik, and M. Maurer, "Scenarios for development, test and validation of automated vehicles," 2018 IEEE Intelligent Vehicles Symposium (IV), pp. 1821-1827, 2018.

[9] T. Menzel, G. Bagschik, L. Isensee, A. Schomburg, and M. Maurer, "From functional to logical scenarios: Detailing a keyword-based scenario description for execution in a simulation environment," 2019 IEEE Intelligent Vehicles Symposium (IV), pp. 2383-2390, 2019.

[10] S. Kitajima, K. Shimono, J. Tajima, J. Antona-Makoshi, and N. Uchida, "Multi-agent traffic simulations to estimate the impact of automated technologies on safety," Traffic Injury Prevention, vol. 20, no. sup1, pp. S58-S64, Jun. 2019.

[11] J. Wang, J. Wu, and Y. Li, "The Driving Safety Field Based on Driver-Vehicle-Road Interactions," IEEE Trans. Intell. Transport. Syst., vol. 16, no. 4, pp. 2203-2214, Aug. 2015.

[12] D. Krajzewicz, "Traffic simulation with sumo - simulation of urban mobility," in Barceló J. (eds) Fundamentals of Traffic Simulation. International Series in Operations Research \& Management Science, vol. 145, June 2010. 
[13] M. Ben-Akiva, H. N. Koutsopoulos, T. Toledo, Q. Yang, C. F. Choudhury, C. Antoniou, and R. Balakrishna, "Traffic Simulation with MITSIMLab," in Barceló J. (eds) Fundamentals of Traffic Simulation. International Series in Operations Research \& Management Science, vol. 145, June 2010, pp. 233-268.

[14] P. A. M. Ehlert and L. J. M. Rothkrantz, "Microscopic traffic simulation with reactive driving agents," in ITSC 2001. 2001 IEEE Intelligent Transportation Systems. Proceedings, 2001, pp. 860-865.

[15] J. Li, A. Doniec, J. Boonaert, and G. Lozenguez, "Which traffic simulator is suitable for customized behaviors in multi-modal scenarios," in 2018 21st International Conference on Intelligent Transportation Systems (ITSC), 2018, pp. 3791-3796.

[16] L. Eboli, G. Mazzulla, and G. Pungillo, "The influence of physical and emotional factors on driving style of car drivers: A survey design," Travel Behaviour and Society, vol. 7, pp. 43 - 51, 2017.

[17] E. Roidl, B. Frehse, and R. Höger, "Emotional states of drivers and the impact on speed, acceleration and traffic violations-a simulator study," Accident Analysis \& Prevention, vol. 70, pp. 282 - 292, 2014.

[18] B. Simms, "Perception and driving: Theory and practice," British Journal of Occupational Therapy, vol. 48, no. 12, pp. 363-366, 1985.

[19] F. P. Mckenna and J. Crick, "Experience and expertise in hazard perception," in Behavioural research in road safety. Proceedings of a seminar held at Nottingham University, 1991, pp. 39-46.

[20] M. Piccione and A. Rubinstein, "On the interpretation of decision problems with imperfect recall," Games and Economic Behavior, vol. 20, no. 1 , pp. $3-24,1997$

[21] — "The absent-minded driver's paradox: Synthesis and responses," Games and Economic Behavior, vol. 20, no. 1, pp. 121 - 130, 1997.

[22] E. Thorn, S. Kimmel, and M. Chaka, "A framework for automated driving system testable cases and scenarios," Washington, DC: National Highway Traffic Safety Administration., Tech. Rep. DOT HS 812 623, 2018.

[23] H. Summala, "Brake reaction times and driver behavior analysis," Transportation Human Factors, vol. 2, pp. 217-226, 2000.

[24] C. Urmson, "Driving beyond stopping distance constraints," in 2006 IEEE/RSJ International Conference on Intelligent Robots and Systems, Oct 2006, pp. 1189-1194.

[25] E. Romera, L. M. Bergasa, and R. Arroyo, "Need data for driver behaviour analysis? presenting the public uah-driveset," 2016 IEEE 19th International Conference on Intelligent Transportation Systems (ITSC), pp. 387-392, 2016.

[26] A. Palazzi, D. Abati, F. Solera, and R. Cucchiara, "Predicting the driver's focus of attention: the dr (eye) ve project," IEEE transactions on pattern analysis and machine intelligence, vol. 41, no. 7, pp. 1720-1733, 2018.

[27] X. Chen, Z. Li, Y. Wang, Z. Cui, C. Shi, and H. Wu, "Evaluating the impacts of grades on vehicular speeds on interstate highways," PLOS ONE, vol. 12, no. 9, pp. 1-15, 092017.

[28] D. Santos, J. Pinto, R. J. F. Rossetti, and E. Oliveira, "Three dimensional modelling of porto's network for electric mobility simulation," in 2016 11th Iberian Conference on Information Systems and Technologies (CISTI), June 2016, pp. 1-6.

[29] E. Andreotti, P. Boyraz, and Selpi, "Supplement: Overtaking scenario from real driving data," https://research.chalmers.se/en/publication/515676, 2020.

[30] T. Liu and Selpi, "Comparison of car-following behavior in terms of safety indicators between china and sweden," IEEE Transactions on Intelligent Transportation Systems, pp. 1-10, 2019.

[31] K. Takeda, J. H. L. Hansen, P. Boyraz, L. Malta, C. Miyajima, and H. Abut, "International large-scale vehicle corpora for research on driver behavior on the road," IEEE Transactions on Intelligent Transportation Systems, vol. 12, pp. 1609-1623, 2011

[32] R. Gallotti, A. Bazzani, and S. Rambaldi, "Understanding the variability of daily travel-time expenditures using gps trajectory data," EPJ Data Science, vol. 4, pp. 1-14, 2015.

[33] D. Tsiotas and S. Polyzos, "The topology of urban road networks and its role to urban mobility," Transportation Research Procedia, vol. 24, pp. 482 - 490, 2017, 3rd Conference on Sustainable Urban Mobility, 3rd CSUM 2016, 26 - 27 May 2016, Volos, Greece.

[34] J. Wang, K. Li, and X.-Y. Lu, "Chapter 5 - effect of human factors on driver behavior," in Advances in Intelligent Vehicles, Y. Chen and L. Li, Eds. Boston: Academic Press, 2014, pp. $111-157$.

[35] D. Procházková, "Chapter 7 - the human factor and its handling," in Advances in Intelligent Vehicles, Y. Chen and L. Li, Eds. Boston: Academic Press, 2014, pp. 199 - 223.

[36] J. Kaths, B. Schott, and F. Chucholowski, "Co-simulation of the virtual vehicle in virtual traffic considering tactical driver decisions," in SUMO User Conference 2019, ser. EPiC Series in Computing, M. Weber,
L. Bieker-Walz, R. Hilbrich, and M. Behrisch, Eds., vol. 62. EasyChair, 2019, pp. 21-28.

[37] M. Semrau and J. Erdmann, "Simulation framework for testing adas in chinese traffic situations," in SUMO2016, ser. Berichte aus dem DLRInstitut für Verkehrssystemtechnik, K. Lemmer, Ed., vol. 30. DLR, May 2016, pp. 103-115.

[38] E. Mintsis, K. Porfyri, L. Luecken, D. Koutras, X. Zhang, M. Rondinone, J. Schindler, S. Maerivoet, L. Akkermans, K. Carlier, I. Mayeres, A. Correa, and E. Mitsakis, "TransAID Deliverable 3.1 - Modelling, simulation and assessment of vehicle automations and automated vehicles' driver behaviour in mixed traffic." 2018.

[39] L. Lücken, E. Mintsis, K. Porfyri, R. Alms, Y.-P. Flötteröd, and D. Koutras, "From Automated to Manual - Modeling Control Transitions with SUMO," in SUMO User Conference 2019, ser. EPiC Series in Computing. EasyChair, 2019, vol. 62, pp. 124-144.

[40] Fredrik Nilsson, "Simulation-based Analysis of Partially Automated Vehicular Networks. A Parametric Analysis Utilizing Traffic Simulation," Master's thesis, Chalmers University of Technology, Gothenburg, Sweden, 2019.

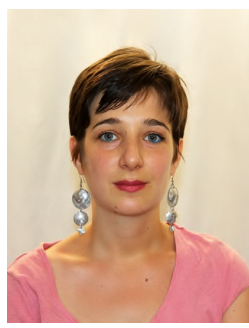

Eleonora Andreotti received BSc and MSc degrees in Mathematics from University of Ferrara and Bologna, respectively, and her $\mathrm{PhD}$ in Mathematics from University of L'Aquila in 2018. After being a visiting student at the Max Planck Insitute for Mathematics in the Sciences of Leipzig from 2017 to 2018 she won a fellowship for a post-doctoral research at the University of Turin in 2018. Currently she is a post-doctoral researcher in Vehicle Safety Division of Mechanics and Maritime Sciences Department at Chalmers University of Technology. Her research activity concerns mathematical modelling, static and dynamical complex systems and spectral graph theory and partitioning.

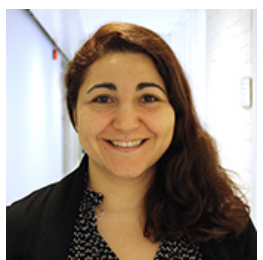

Pinar Boyraz (M' 2009, SM' 2019) received double-major BSc. degrees in mechanical and textile engineering from Istanbul Technical University (ITU), TR in 2003-2004 and her PhD in Mechatronics from Wolfson School of Mechanical and Manufacturing Engineering, Loughborough University, UK in 2008. She worked as a Post-doctoral RA in the Erik Jonsson School of Engineering and Computer Science, University of Texas at Dallas, in U.S.A. during 2008-2010, focusing on driver behavior modeling and active safety system development. She was an Assist. Prof. during 2010-2014 and an Assoc. Prof. during 2014-2018 in Mechanical Engineering Dept. of ITU, TR, conducting research in applied robotics. She was awarded by Alexander von Humboldt Foundation with Experienced Researcher Fellowship during her project in applied robotics at Leibniz University of Hannover, Germany in 2016-17. Since March 2018, she works as an Assoc. Prof. at Mechanics and Maritime Sciences Dept. of Chalmers University of Technology, Gothenburg, Sweden. Her research interests broadly include applications of mathematical modelling, mechatronics, signal processing and control theory.

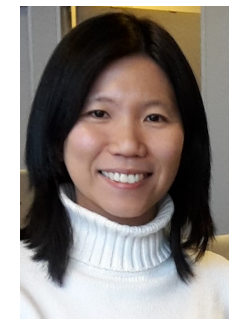

Selpi received a $\mathrm{PhD}$ degree in computing from the Robert Gordon University in the UK in 2008, an MSc degree in bioinformatics from Chalmers University of Technology in Sweden in 2004, and a BSc degree in computer science from the University of Indonesia in 2000. She currently works at Chalmers University of Technology. Her current research interest is on understanding how mixed traffic, with vehicles with different driving styles and automation levels sharing the same roads, affects traffic safety and efficiency. She is also interested in applications of machine learning and data mining for transport domain (e.g., understanding driving styles/driver behaviour from naturalistic driving data, travel time and traffic volume predictions, text-mining for text data in transport). Beside academic work, she has several years of experiences in software industry. Dr. Selpi is a member of the IEEE Intelligent Transportation Systems Society's technical committee on Naturalistic Driving Data Analytics. She has served as a reviewer and an associate editor for several IEEE conferences. 